\title{
Adiponectin suppresses inflammatory responses at the early phase of atherosclerosis in hyperglycemic rats
}

\author{
JIN-BO LIU ${ }^{1,3}$, LIN JIA ${ }^{2}$, BING-RONG LI ${ }^{3}$, LI-ZHEN LAN ${ }^{3}$, \\ QIAN GE ${ }^{3}$, HONG-TING ZHENG ${ }^{3}$ and HUA-CONG DENG ${ }^{3}$

\begin{abstract}
Departments of ${ }^{1}$ Endocrinology, and ${ }^{2}$ Obstetrics and Gynecology, Qilu Hospital, Shandong University, Jinan; ${ }^{3}$ Department of Endocrinology, The First Affiliated Hospital, Chongqing Medical University, Chongqing, P.R. China
\end{abstract}

Received November 6, 2009; Accepted December 21, 2009

DOI: 10.3892/mmr_00000260

\begin{abstract}
Adiponectin plays an important role in atherosclerosis, but its relationship with the early initiation of atherosclerosis in diabetes mellitus is still not completely understood. In this study, we defined the role of adiponectin early in the process of atherosclerosis in hyperglycemic rats. Recombinant adenovirus expressing the full-length apM1 cDNA gene (Ad-APN) was constructed and successfully transfected into hyperglycemic rats characterized by the presentation of early atherosclerosis. The levels of sICAM-1 and C-reactive protein (CRP) in serum as well as the expression of VCAM-1, ICAM-1 and MCP-1 in aortic tissue were evaluated. Serum adiponectin was significantly increased in Ad-APN-treated rats compared with Ad- $\beta$ gal-treated rats. The levels of sICAM- 1 and CRP in serum were dramatically reduced by 22 and $21 \%$, respectively, in Ad-APN-treated rats. Additionally, in aortic tissue, significantly reduced mRNA levels of VCAM-1, ICAM-1 and MCP-1 were observed after Ad-APN transfection. These results suggest that, in hyperglycemic rats, adiponectin plays an inhibitory role in the early development of atherosclerosis. In conclusion, the protective effect of adiponectin is associated with the reduced activity of various inflammation-related factors.
\end{abstract}

\section{Introduction}

Adiponectin, also called GBP28, ACRP30 or AdipoQ, is mainly expressed and secreted in adipose tissue. Previous clinical studies have demonstrated that low levels of plasma adiponectin (hypoadiponectinemia) are strongly correlated with atherosclerotic cardiovascular diseases (1-4). Further insights have revealed that adiponectin inhibits the progression of atherosclerosis through anti-atherogenic effects, both in vitro and in vivo. Detailed studies carried out on signaling

Correspondence to: Professor Huacong Deng, Department of Endocrinology, The First Affiliated Hospital, Chongqing Medical University, Youyi Road, Chongqing 400016, P.R. China

E-mail: ljbsdu@gmail.com

Key words: adiponectin, atherosclerosis, hyperglycemia, inflammation mechanisms showed that, in cultured human aortic endothelial cells, physiological concentrations of human recombinant adiponectin reduced the expression of tumor necrosis factor- $\alpha$ related adhesion molecules, the proliferation of vascular smooth muscle cells and, furthermore, the transformation of macrophages to foam cells via increased crosstalk between the cAMP-PKA and NF- $\kappa \mathrm{B}$ signaling pathways (5). Adiponectin, which is highly detected in fatty streak lesions composed of macrophages and foam cells, was found to suppress the mRNA levels of vascular cell adhesion molecule-1 (VCAM-1) and class A scavenger receptor in apoE $\mathrm{E}^{-/}$mice (6). This is the first report to suggest that adiponectin exerts an inhibitory effect on the development of atherosclerosis in vivo.

It is widely accepted that the atherosclerotic process is initiated and aggravated by endothelial dysfunction. At the early stage of atherosclerosis, endothelial cell activation by various inflammatory stimuli results in enhanced expression of adhesion molecules on the endothelial surface. These molecules further contribute to the increased activity of leukocyte adhesion and transendothelial migration, which is accompanied by extravasations of low-density lipoprotein (LDL) into the vessel wall, subsequent foam cell and plaque formation, and eventually cell necrosis. Disruptions to the structures of these damaged endothelial cells, such as the adherens membrane junction, facilitate the transportation of pro-atherosclerotic compounds into the vessel wall.

Atherosclerosis accounts for $65-80 \%$ of all diabetes-related deaths among North Americans, and individuals with diabetes have a 2- to 4-fold inceased risk of mortality from coronary artery disease (CAD). Furthermore, when patients with atherosclerosis develop diabetes, they have a worse prognosis than those without diabetes. The American Heart Association has stated that 'diabetes is a cardiovascular disease', emphasizing that cardiovascular disease is frequently attributed to diabetes. Despite the interest in the relationship between atherosclerosis and diabetes, few studies have focused on the early stage of atherosclerosis in the diabetic state. Hyperglycemia remains the primary characteristic of diabetes mellitus. Thus, in order to obtain new insights into the underlying mechanisms involved in the early development of atherosclerosis, the present study aimed to clarify whether increased serum adiponectin suppresses inflammatory responses at the early phase of atherosclerosis in hyperglycemic rats. 
Table I. Primers for RT-PCR and real-time PCR amplification.

\begin{tabular}{|c|c|c|c|}
\hline Gene & Primer sequence $\left(5^{\prime} \rightarrow 3^{\prime}\right)$ & $\mathrm{T}_{\mathrm{m}}\left({ }^{\circ} \mathrm{C}\right)$ & Length (bp) \\
\hline \multirow[t]{2}{*}{ VCAM-1 } & F: CGGTCATGGTCAAGTGTTTG & 55.4 & \multirow[t]{2}{*}{570} \\
\hline & R: GAGATCCAGGGGAGATGTCA & 57.4 & \\
\hline \multirow{2}{*}{ ICAM-1 } & F: CAAACGGGAGATGAATGG & 52.1 & \multirow{2}{*}{183} \\
\hline & R: TGGCGGTAATAGGTGTAAAT & 51.3 & \\
\hline \multirow[t]{2}{*}{ GAPDH } & F: AGGTTGTCTCCTGTGACTTCA & 54.1 & \multirow[t]{2}{*}{184} \\
\hline & R: TGGTCCAGGGTTTCTTACTCC & 58.2 & \\
\hline \multirow[t]{2}{*}{$\beta$-actin } & F: GAGAGGGAAATCGTGCGTGAC & 59.8 & \multirow[t]{2}{*}{453} \\
\hline & R: CATCTGCTGGAAGGTGGACA & 57.4 & \\
\hline
\end{tabular}

\section{Materials and methods}

Recombinant adenovirus. Full-length apM1 cDNA coding sequences were derived from human adipose tissue using TaqMan Reverse Transcription kits (Qiagen, Germany). The replication-defective recombinant adenovirus was constructed with the AdEasy ${ }^{\mathrm{TM}}$ Adenoviral Vector System (Q-Biogene, USA). An adenovirus expressing the full-length apM1 cDNA gene (Ad-APN) or $\beta$-galactosidase gene (Ad- $\beta$ gal) was propagated in HEK 293 cells, purified by $\mathrm{CsCL}$ gradient centrifugations and stored at $-80^{\circ} \mathrm{C}$ until use.

Animal protocol. Experimental procedures were approved by the Animal Experimentation Ethics Committee (Chongqing Medical University, China), and were in accordance with the National Health and Medical Research Council of China Guidelines on Animal Experimentation. Eight-week-old male Wistar rats weighing $206.35 \pm 12.48 \mathrm{~g}$ were housed under controlled temperature conditions and a 12-h light/dark cycle. Two different diets were used in this study: an 'atherogenic' diet known to elicit hyperlipidemia in Wistar rats containing $10 \%$ fat, $3 \%$ cholesterol and $0.5 \%$ sodium cholate, and a standard rodent diet containing $2.2 \%$ fat, $15 \%$ protein and $5.2 \%$ crude fiber. Hyperglycemia was induced by an intraperitoneal injection of streptozotocin (30 g/kg) (Sigma-Aldrich, USA) in rats that had been fed an 'atherogenic' diet for 4 weeks. After $72 \mathrm{~h}$ of streptozotocin administration, blood was collected from the tail vein for glucose analysis. Animals showing fasting blood glucose $>300 \mathrm{mg} / \mathrm{dl}(16.7 \mathrm{mmol} / \mathrm{l})$ were considered to be hyperglycemic, and were maintained on an 'atherogenic' diet for another 12 weeks. At the age of 14 weeks, Ad-APN and Ad- $\beta$ gal was injected into the tail vein $\left(2 \times 10^{8} \mathrm{pfu}\right.$ each) of the mice. Finally, serum was collected, and the animals were sacrificed. Hepatic and aortic tissue was kept for subsequent investigations. H\&E staining was used to confirm the presence of atherosclerosis.

Serum analysis. Blood samples were collected 2 weeks after adenovirus injection. Total cholesterol (TC), triglycerides (TG), high-density lipoprotein cholesterol (HDL-C) and lowdensity lipoprotein cholesterol (LDL-C) were measured with enzymatic kits (Biosino, China). Additionally, ELISA was used to determine the levels of plasma adiponectin (Innogent, China), soluble intercellular adhesion molecule-1 (sICAM-1)
(BPB Biomedicals, USA) and C-reactive protein (CRP) (DiaSys, Germany).

Total RNA isolation, RT-PCR and real-time PCR. Total RNA was isolated from rat aortic tissue with TRIzol reagent (Invitrogen, USA) according to the manufacturer's protocol. RNA concentrations were measured at A260 with a Beckman DU7500 UV/Vis spectrophotometer. The integrity of RNA was verified by electrophoresis. cDNA was synthesized from the total RNA by reverse transcription reaction with Takara RNA PCR kits (AMV) ver. 3.0 (Takara, China). Reverse transcribed products were kept for RT-PCR and real-time PCR. The specific primers involved are summarized in Table I.

To investigate the expression of VCAM-1 in the rats, RT-PCR was performed with the Takara RNA PCR kit, yielding a product of $570 \mathrm{bp}$. RT-PCR conditions consisted of an initial denaturation step at $99^{\circ} \mathrm{C}$ for $5 \mathrm{~min}$, followed by 30 cycles at $94^{\circ} \mathrm{C}$ for $30 \mathrm{sec}$, annealing at $53^{\circ} \mathrm{C}$ for $30 \mathrm{sec}$ and extension at $72^{\circ} \mathrm{C}$ for $60 \mathrm{sec}$. Amplified PCR products were then electrophoresed on $1.2 \%$ agarose gel and visualized with ethidium bromide under UV light. $\beta$-actin was amplified as a reference for the quantification of VCAM-1 mRNA.

To quantify the expression of ICAM-1 and monocyte chemoattractant protein-1 (MCP-1) in rat aortic tissue, realtime PCR was carried out using SYBR Premix Ex Taq ${ }^{\mathrm{TM}}$ kits (Takara) on a Rotor-Gene ${ }^{\mathrm{TM}} 3000$ (Corbett Life Science, Australia). The PCR reactions were initiated with a total volume of $25 \mu$ l, including $12.5 \mu \mathrm{l}$ SYBR Premix Ex Taq, $0.5 \mu \mathrm{l}$ forward and reverse primers, $2 \mu \mathrm{l} \mathrm{cDNA}$ samples and $10 \mu \mathrm{l}$ water, followed by $95^{\circ} \mathrm{C}$ for $60 \mathrm{sec}$, then 40 cycles of $95^{\circ} \mathrm{C}$ for $15 \mathrm{sec}, 56^{\circ} \mathrm{C}$ (ICAM- 1 ) or $59^{\circ} \mathrm{C}$ (MCP- 1 and GAPDH) for $15 \mathrm{sec}$, and a final extension at $72^{\circ} \mathrm{C}$ for $20 \mathrm{sec}$. GAPDH was used as a vehicle control to normalize the expression of ICAM-1 and MCP-1. Real-time PCR experiments were run in triplicate. Water was used as a negative control. Data were analyzed by software version 6.0 (Corbett Life Science).

Western blotting. To evaluate the changes in the protein levels of VCAM-1 in the aortic tissue, Western blotting was performd. Freshly frozen aortic tissues were lysed with RIPA buffer $(50 \mathrm{mM}$ Tris- $\mathrm{HCl} \mathrm{pH} 7.4,150 \mathrm{mM} \mathrm{NaCl}, 1 \% \mathrm{NP} 40$, $0.5 \%$ dexycholic acid, sodium and $0.1 \%$ SDS). Proteins were resolved on an $8 \%$ SDS-PAGE gel prior to transfer to polyvinylidene fluoride (PVDF) membranes. The blots were then 


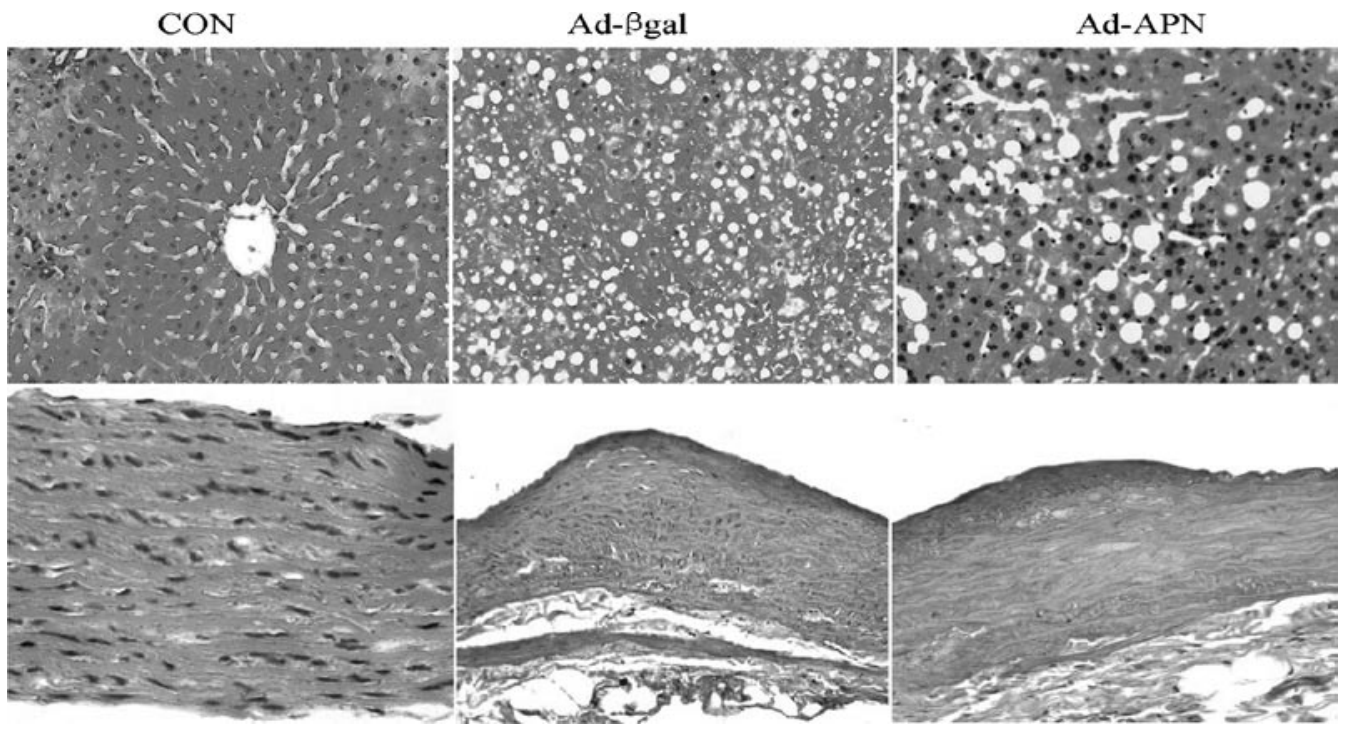

Figure 1. Representative H\&E staining in the liver and aortic tissues of male Wistar rats. The upper panel shows the fatty degeneration of hepatocytes in Ad-Bgal- and Ad-APN-treated rats, which was characterized by the appearance of a large lipid vacuole in hepatocytes and a shift in the nucleus to the cell wall (x100). The lower panel shows the pathological changes at the early stage of atherosclerosis in Ad-ßgal- and Ad-APN-treated rats. A typical intimal thickening, proliferated and disorganized medial smooth muscle cells, and internal elastic lamina with irregular and sawtooth margins were observed in contrast to the

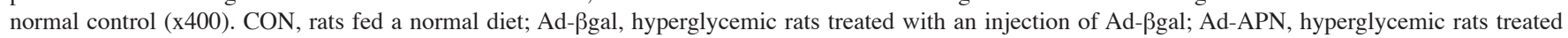
with an injection of Ad-APN.

Table II. Correlation analysis between adiponectin and related factors.

\begin{tabular}{lcc}
\hline Adiponectin & r-value & P-value \\
\hline TC & -0.363 & $<0.010$ \\
TG & -0.483 & 0.042 \\
HDL-C & 0.439 & 0.001 \\
LDL-C & -0.655 & 0.003 \\
CRP & -0.349 & 0.001 \\
sICAM-1 & -0.324 & 0.073 \\
FBG & -0.278 & 0.068 \\
\hline
\end{tabular}

TC, total cholesterol; TG, triglycerides; HDL-C, high-density lipoprotein cholesterol; LDL-C, low-density lipoprotein cholesterol; CRP, C-reactive protein; sICAM-1, soluble intercellular adhesion molecule-1; FBG, fasting blood glucose.

blocked in $5 \%$ non-fat milk overnight at $4^{\circ} \mathrm{C}$. Membranes with transferred proteins were incubated with primary antibodies against VCAM-1 (1:400, goat polyclonal antibody; Santa Cruz, USA) for $2 \mathrm{~h}$ at room temperature, followed by exposure to a secondary antibody at 1:4000 dilution for $1 \mathrm{~h}$ at room temperature. Anti- $\beta$-actin was used as a control. The blots were further developed using chemiluminescence reagent. Densitometric analysis of the scanned images was perfomed using Software Image Quant.

Statistical analysis. Data obtained were analyzed with one-way ANOVA or the Mann-Whitney U test and presented as the mean $\pm \mathrm{SD}$. A P-value $<0.05$ was considered statistically significant.

\section{Results}

Alterations in morphology and metabolic parameters in hyperglycemic rats. Hyperglycemia was successfully induced in rats receiving an 'atherogenic' diet and intraperitoneal injections of streptozotocin for 16 weeks. H\&E staining in the liver and aortic tissue showed the appearance of fatty degeneration of hepatocytes and pathological changes at the early stage of atherosclerosis in contrast to the control group. In addition, typical arterial atheromatous plaque was not detected in the hyperglycemic rats (Fig. 1). Metabolic parameters in the hyperglycemic rats are summarized in Fig. 2A-C. In the hyperglycemic rats compared to the normal control, the concentrations of fasting blood glucose (FBG), $\mathrm{TC}$, TG and LDL-C were significantly increased $(\mathrm{P}<0.01)$, while the concentrations of rat adiponectin and HDL-C were decreased $(\mathrm{P}<0.05)$. The results of the correlation analysis showed that the levels of serum adiponectin were negatively correlated with TC, TG, LDL-C and CRP $(\mathrm{P}<0.05)$, and positively correlated with HDL-C $(\mathrm{P}<0.01)$. However, no significant correlation with sICAM-1 was observed. Detailed data are presented in Table II.

Effects of elevated serum adiponectin levels on rat body weight, metabolic parameters and atherosclerotic lesions. Competition ELISA was used to detect the concentration of serum endogenous adiponectin. As shown in Fig. 2A, the concentration of serum rat adiponectin in the hyperglycemic rats was lower than that in the normal rats $(\mathrm{P}<0.01)$, while no significant difference was observed between Ad- $\beta$ galand Ad-APN-treated hyperglycemic rats. After 14 days of adenovirus injection, the level of serum human adiponectin encoded by the adenovirus was $68.4 \pm 19.2 \mu \mathrm{g} / \mathrm{ml}$ in Ad-APNtreated rats measured by sandwich ELISA, but no significant 
A

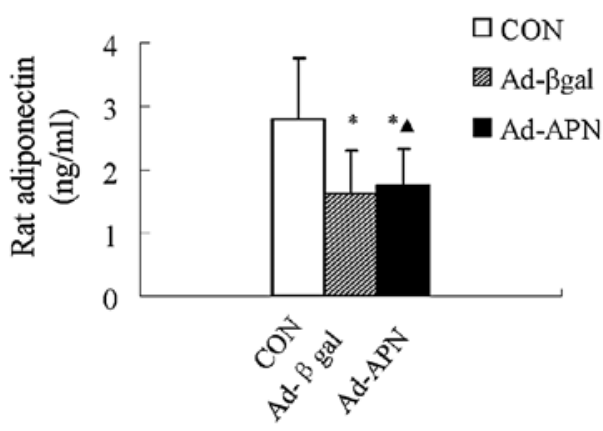

C

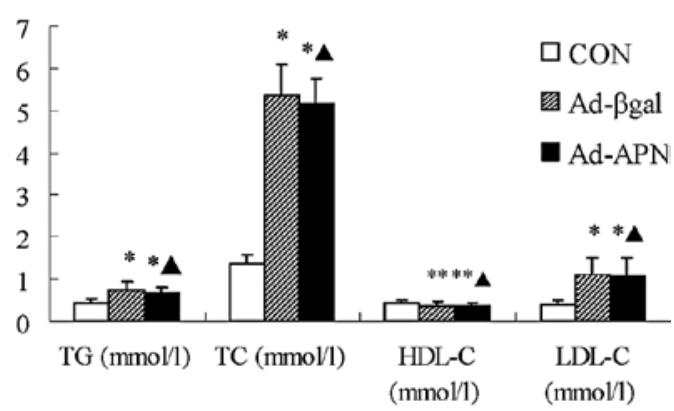

B

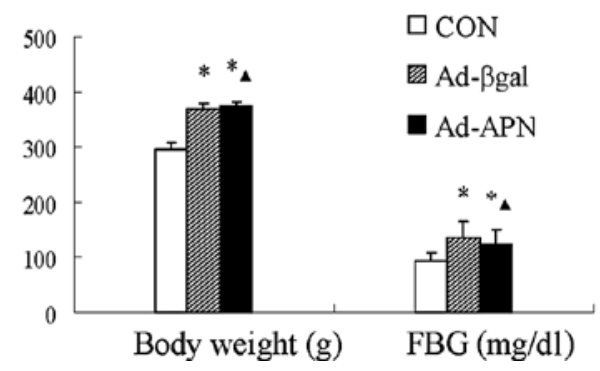

D

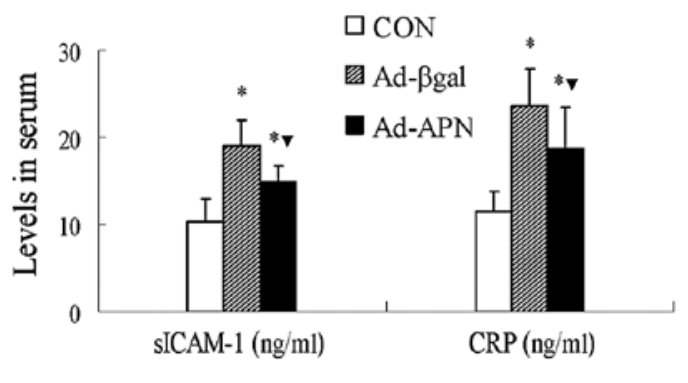

Figure 2. Effects of elevated adiponectin on the values of body weight, fasting blood glucose (FBG), various metabolic markers, sICAM-1 and C-reactive protein (CRP) in rats. (A) Concentration of serum rat adiponectin; (B) alternations in body weight and FBG; (C) concentrations of triglycerides (TG), total cholesterol (TC), high-density lipoprotein cholesterol (HDL-C) and low-density lipoprotein cholesterol (LDL-C); (D) levels of serum sICAM-1 and CRP in Ad- $\beta$ gal- and Ad-APN-treated rats. Male Wistar rats fed a standard rodent diet served as the control. ${ }^{*} \mathrm{P}<0.01,{ }^{* *} \mathrm{P}<0.05$ compared to the corresponding control. $\checkmark \mathrm{P}<0.01, \triangleleft \mathrm{P}>0.05$, Ad-Bgal- compared Ad-APN-treated rats. Data are presented as the mean $\pm \mathrm{SD}$ of three independent experiments.

A
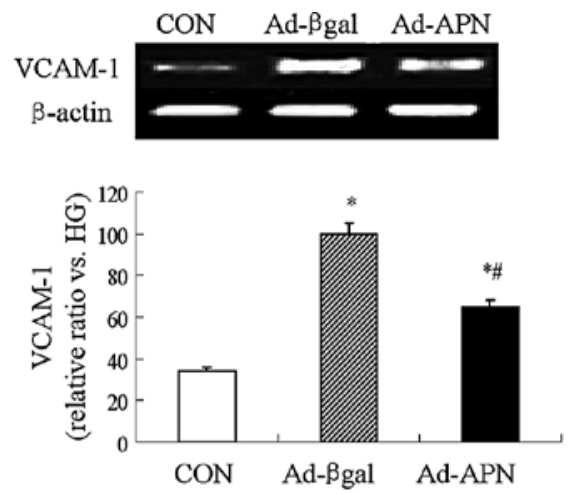

C

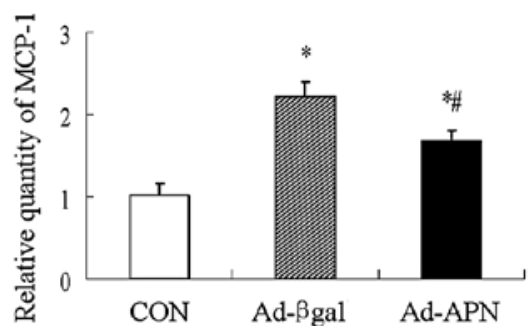

B
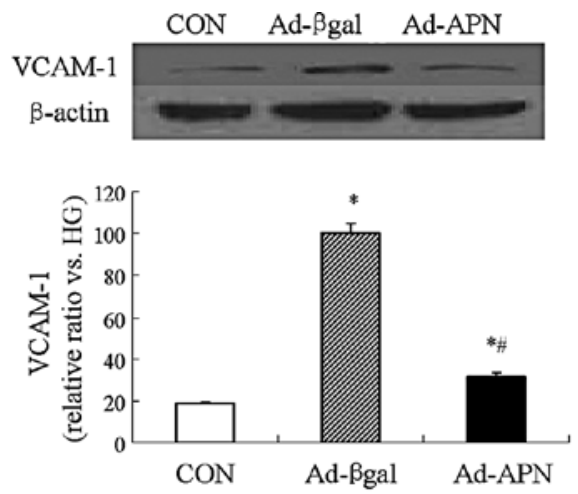

D

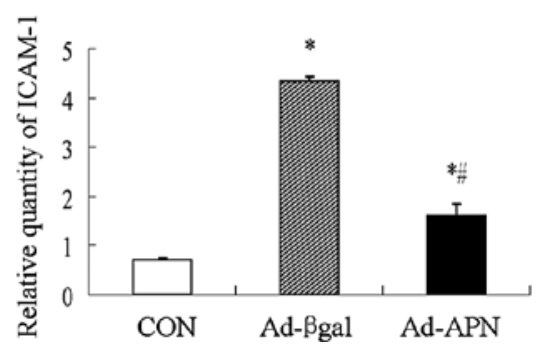

Figure 3. Effects of elevated serum adiponectin on the expression of VCAM-1, ICAM-1 and MCP-1 in aortic tissue. (A) Representative RT-PCR images and the corresponding densitometric results indicate the expression of VCAM-1 mRNA in Ad- $\beta$ gal- and Ad-APN-treated rats. (B) Consistent with the expression of mRNA, Western blotting indicates the protein level of VCAM-1 in Ad-ßgal- and Ad-APN-treated rats. Expression of (C) MCP-1 and (D) ICAM-1 mRNA

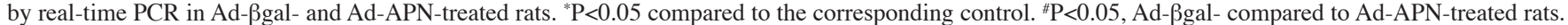
Data are presented as the mean $\pm \mathrm{SD}$ of three independent experiments. $\beta$-actin served as an internal normalization control.

difference was observed between the Ad- $\beta g a l$ and Ad-APN groups in terms of body weight (BW), FBG, TC, TG, HDL-C and LDL-C (Fig. 2B and C). Compared with the Ad- $\beta$ galtreated rats, pathological changes were not obviously improved in the Ad-APN-treated hyperglycemic rats.
Effects of elevated serum adiponectin on the levels of ICAM-1 and CRP in serum. The concentrations of serum SICAM-1 and CRP were tested by ELISA. As shown in Fig. 2D, the levels of serum sICAM-1 and CRP were much higher in the Ad- $\beta$ gal-treated rats than in the control rats $(\mathrm{P}<0.01)$. When 
compared with the Ad- $\beta$ gal-treated rats, the Ad-APN-treated hyperglycemic rats, which were administered the adenovirus injection for 14 days, presented with a pronounced reduction in serum sICAM-1 and CRP of $22 \%(\mathrm{P}<0.01)$ and $21 \%(\mathrm{P}<0.01)$, respectively (Fig. 3).

Effects of elevated serum adiponectin on the expression of VCAM-1, ICAM-1 and MCP-1 in aortic tissue. To further determine the anti-inflammatory property of adiponectin, we examined the expression of VCAM-1, ICAM-1 and MCP-1 in aortic tissue by RT-PCR, real-time PCR and Western blotting. In contrast to the normal control, the expression of VCAM-1, ICAM-1 and MCP-1 in aortic tissue was significantly increased in the Ad- $\beta$ gal-treated rats $(\mathrm{P}<0.05)$. As demonstrated in Fig. $3 \mathrm{~A}$ and $\mathrm{B}$, the increment of adiponectin in the Ad-APN-treated rats compared to the Ad- $\beta$ gal-treated rats resulted in a clear reduction in VCAM-1 expression of $\sim 30 \%$ at both the mRNA and protein levels $(\mathrm{P}<0.05)$. We also investigated the expression of ICAM-1 and MCP-1. Consistent with the tendency of VCAM-1, the expression of ICAM-1 and MCP-1 was significantly suppressed by 63 and 25\% $(\mathrm{P}<0.05)$, respectively (Fig. 3C and D).

\section{Discussion}

In this study, hyperglycemia in rats was successfully induced by the intraperitoneal injection of streptozotocin, and was characterized by hyperglycemia, dyslipidemia and pro-inflammatory states. Therefore, these hyperglycemic rats were considered to be predisposed to atherosclerosis. Results from H\&E staining revealed the appearance of fatty degeneration of hepatocytes in hyperglycemia rats, but typical arterial atheromatous plaque was not identified. Additional pathological changes including intimal thickening, proliferated and disorganized medial smooth muscle cells; internal elastic lamina with an irregular as well as sawtooth margin were also detected in the lesions of the hyperglycemic rats. Accordingly, the rats were determined to be at the early stage of atherosclerosis. In the Ad-APNtreated hyperglycemic rats, such pathological changes were not noticeably improved.

Previous studies have shown that, among different ethnic groups, CAD, hypertension, obesity, type 2 diabetes, arteriosclerosis obliterans and ischemic heart disease are associated with reduced levels of adiponectin in serum (3,7-9). Moreover, adiponectin concentrations are significantly lower in male patients with both CAD and type 2 diabetes than in those with diabetes alone (5). Consistent with these reports, in the present study the hyperglycemic rats had significantly lower serum adiponectin concentrations than the control rats. Further correlation analysis showed that the levels of serum adiponectin were negatively correlated with TC, TG, LDL-C and CRP and positively correlated with HDL-C. High-sensitive CRP (hs-CRP) is a well-known risk factor for CAD and a sensitive marker of inflammation. An inverse relationship has been observed between circulating concentrations of CRP and adiponectin in diabetic patients (10-13). Based on these findings, we proposed that low levels of circulating adiponectin serve as a possible predictor of the pro-inflammatory state in hyperglycemic rats.

Patients with diabetes mellitus have a markedly increased incidence of atherosclerosis, which is largely caused by an increased activity of inflammation (14). Endothelial injury, mediated by various inflammatory stimuli, is responsible for the formation of local atherosclerotic lesions at the early stage of the disease. Since studies regarding the condition of early atherosclerosis in diabetes are few, this study investigated the effect of adiponectin on the expression of inflammatory factors in the vascular endothelium.

The endothelium is well known for its resistance to leukocyte adhesion, but these anti-adhesive properties might be damaged by hypercholesterolemia. Oxidized LDL and angiotensin II increase the production of pro-inflammatory cytokines including MCP-1, ICAM-1 and VCAM-1 $(15,16)$. Endothelial alterations coupled with elevated CRP levels set the stage for the initiation and progression of atherogenesis by promoting inflammation within the vessel wall (17). In the present study conducted in hyperglycemic rats, elevated levels of CRP and sICAM-1 in circulation as well as of MCP-1, VCAM-1 and ICAM-1 in aortic tissue were observed, suggesting that endothelial dysfunction and inflammatory changes arose at the early stage of atherosclerosis in the hyperglycemic rats.

Previous studies have shown that adiponectin knockout (KO) in mice accelerates intimal proliferation in mechanically injured vascular walls. Adiponectin KO mice infected by Ad-APN prior to vascular injury exhibited a 2- to 3-fold increase in serum levels of adiponectin, leading to less intimal thickening (18). Adipo (-/-) mice exhibited an excess production of key chemoattractant cytokines and increased expression of the aortic endothelial adhesion molecules VCAM-1 and ICAM-1 (19). Here, for the first time, we demonstrated that the adenovirus-mediated increase of serum adiponectin inhibited the expression of ICAM-1, VCAM-1 and MCP-1 in aortic tissue, and suppressed the levels of sICAM-1 and CRP in serum in hyperglycemic rats. Devaraj et al (20) demonstrated that adiponectin reduced CRP synthesis and secretion from human aortic endothelial cells under hyperglycemia via the up-regulation of AMP kinase and the down-regulation of $\mathrm{NF}-\kappa \mathrm{B}$. Although the effect of adiponectin on plaque status was not observed, some studies have shown that adiponectin was capable of reducing the plaque area in atherosclerosis $(6,21)$. These findings are indicative of the anti-inflammatory role of adiponectin. To exclude the possible effect generated by endogenous adiponectin, we compared adiponectin expression between Ad- $\beta$ gal- and Ad-APN-treated rats. No significant difference was observed, which in turn indicates that human adiponectin encoded by an adenovirus exhibits anti-inflammation activity, and eventually hinders the development of atherosclerosis.

In summary, adiponectin has anti-inflammatory properties and suppresses the initiation and progression of atherosclerosis in hyperglycemic rats, particularly at the early stage without atheromatous plaques. Conversely, hypoadiponectinemia is indicative of dyslipidemia and a pro-inflammatory state, paralleled with increased levels of ICAM-1, VCAM-1, MCP-1 and CRP. Hence, the findings of this study provide promising potential targets for the prevention of the initiation and progression of atherosclerosis in patients with diabetes mellitus.

\section{Acknowledgements}

This work was supported by grants from the National Natural Science Foundation of China (no. 30370670) to Hua-Cong Deng. 


\section{References}

1. Cavusoglu E, Ruwende C, Chopra V, et al: Adiponectin is an independent predictor of all-cause mortality, cardiac mortality and myocardial infarction in patients presenting with chest pain. Eur Heart J 27: 2300-2309, 2006.

2. Koenig W, Khuseyinova N, Baumert J, Meisinger C and Lowel H: Serum concentrations of adiponectin and risk of type 2 diabetes mellitus and coronary heart disease in apparently healthy middle-aged men: results from the 18-year follow-up of a large cohort from southern Germany. J Am Coll Cardiol 48: 1369-1377, 2006.

3. Iwashima Y, Horio T, Suzuki Y, et al: Adiponectin and inflammatory markers in peripheral arterial occlusive disease. Atherosclerosis 188: 384-390, 2006.

4. Yaturu S, Bridges JF and Subba Reddy DR: Decreased levels of plasma adiponectin in prediabetes, type 2 diabetes and coronary artery disease. Med Sci Monit 12: CR17-CR20, 2006.

5. Ouchi N, Kihara S, Arita Y, et al: Adiponectin, an adipocytederived plasma protein, inhibits endothelial NF-kappaB signaling through a cAMP-dependent pathway. Circulation 102: 1296-1301, 2000.

6. Okamoto Y, Kihara S, Ouchi N, et al: Adiponectin reduces atherosclerosis in apolipoprotein E-deficient mice. Circulation 106: 2767-2770, 2002.

7. Weyer C, Funahashi T, Tanaka S, et al: Hypoadiponectinemia in obesity and type 2 diabetes: close association with insulin resistance and hyperinsulinemia. J Clin Endocrinol Metab 86 1930-1935, 2001

8. Dzielinska Z, Januszewicz A, Wiecek A, et al: Decreased plasma concentration of a novel anti-inflammatory protein - adiponectin - in hypertensive men with coronary artery disease. Thromb Res 110: 365-369, 2003.

9. Kawano T, Saito T, Yasu T, et al: Close association of hypoadiponectinemia with arteriosclerosis obliterans and ischemic heart disease. Metabolism 54: 653-656, 2005.

10. Shetty GK, Economides PA, Horton ES, Mantzoros CS and Veves A: Circulating adiponectin and resistin levels in relation to metabolic factors, inflammatory markers and vascular reactivity in diabetic patients and subjects at risk for diabetes. Diabetes Care 27: 2450-2457, 2004.
11. Yuan G, Zhou L, Tang J, et al: Serum CRP levels are equally elevated in newly diagnosed type 2 diabetes and impaired glucose tolerance and related to adiponectin levels and insulin sensitivity. Diabetes Res Clin Pract 72: 244-250, 2006.

12. Mojiminiyi OA, Abdella NA, Al Arouj M and Ben Nakhi A: Adiponectin, insulin resistance and clinical expression of the metabolic syndrome in patients with type 2 diabetes. Int $\mathbf{J}$ Obes 31: 213-220, 2007.

13. Dullaart RP, De Vries R, Sluiter WJ and Voorbij HA: High plasma C-reactive protein is related to low paraoxonase-I activity independently of high leptin and low adiponectin in type 2 diabetes mellitus. Clin Endocrinol 70: 221-226, 2008.

14. Ross R: Atherosclerosis - an inflammatory disease. N Engl J Med 340: 115-126, 1999.

15. Cybulsky MI and Gimbrone MA Jr: Endothelial expression of a mononuclear leukocyte adhesion molecule during atherogenesis. Science 251: 788-791, 1991

16. Kranzhofer R, Schmidt J, Pfeiffer CA, Hagl S, Libby P and Kubler W: Angiotensin induces inflammatory activation of human vascular smooth muscle cells. Arterioscler Thromb Vasc Biol 19: 1623-1629, 1999.

17. Verma $\mathrm{S}$, Wang $\mathrm{CH}$, Li SH, et al: A self-fulfilling prophecy: C-reactive protein attenuates nitric oxide production and inhibits angiogenesis. Circulation 106: 913-919, 2002.

18. Matsuda M, Shimomura I, Sata M, et al: Role of adiponectin in preventing vascular stenosis. The missing link of adipo-vascular axis. J Biol Chem 277: 37487-37491, 2002.

19. Teoh H, Quan A, Bang KW, et al: Adiponectin deficiency promotes endothelial activation and profoundly exacerbates sepsis-related mortality. Am J Physiol Endocrinol Metab 295: E658-E664, 2008.

20. Devaraj S, Torok N, Dasu MR, Samols D and Jialal I: Adiponectin decreases C-reactive protein synthesis and secretion from endothelial cells: evidence for an adipose tissue-vascular loop. Arterioscler Thromb Vasc Biol 28: 1368-1374, 2008.

21. Li CJ, Sun HW, Zhu FL, et al: Local adiponectin treatment reduces atherosclerotic plaque size in rabbits. J Endocrinol 193: 137-145, 2007. 